\title{
Do Demographic and Disease Structures Affect the Recurrence of Epidemics?
}

\author{
A. Castellazzo, A. Mauro, C. Volpe, E. Venturino* \\ Dipartimento di Matematica "Giuseppe Peano" Università di Torino \\ via Carlo Alberto 10, 10123 Torino, Italy
}

\begin{abstract}
In this paper we present an epidemic model affecting an age-structured population. We show by numerical simulations that this demographic structure can induce persistent oscillations in the epidemic. The model is then extended to encompass a stage-structured disease within an age-dependent population. In this case as well, persistent oscillations are observed in the infected as well as in the whole population.
\end{abstract}

Keywords and phrases: age-structured population, stage-structured epidemics

Mathematics Subject Classification: 92D25, 92D30

\section{Introduction}

The problem we want to address here is a generalization of the classical epidemic model. This is achieved via the introduction of age- and stage-dependencies in the population. This model has been first presented in [8], showing its well posedness, namely the existence and uniqueness of solutions. In [9], the idea of a disease progressing in stages has been extended to ecoepidemic models, which describe interacting populations among which a disease spreads. For an introduction on this field of research see for instance $[2,6,10]$.

A nice review of epidemiology is contained in [3]. To model diseases spreading in a population, we partition the whole population into at least three distinct classes: susceptible individuals, i.e. those that are healthy and can therefore contract the disease, infected individuals, who contracted the disease but have not yet been recognized as disease-carriers, and removed individuals, who are the ones in which the disease has been detected and for which measures are taken, so that they do not spread it any further. The purpose of this paper is to introduce two additional characteristics in the description of the situation, to make such a general model more realistic. These features are represented by an age structure in the population, to better distinguish individuals that are more prone to be subject to the disease, and by a stage dependency for the disease in order to take into account the evolution of the illness. Introduction of the stage-dependency requires that a new parameter be specified. For an infected individual, it counts the units of time since contagion has occurred. At the same time, for an individual in the class of removed, it can describe the stage in the recovery period. Building all these features into a model requires the use

${ }^{*}$ Corresponding author. E-mail: ezio.venturino@unito.it 
of hyperbolic partial differential equations. Furthermore, the resulting system is highly nonlinear. Since this renders the theoretical analysis extremely tough, to understand the solution's behavior we perform numerical simulations. The latter reveal that in suitable circumstances such models exhibit coexistence via limit cycles.

The paper is organized as follows. At first, stage dependency is ignored. Thus the model is described via densities of the three classes in which the population is divided, which are functions of time and age only. This is analyzed in Section 2. In Section 3 we study in detail the complete model, including also the stage of the disease. In this case mathematically the infected and removed individuals become functions of three variables. A final discussion concludes the paper.

\section{The age-dependent epidemic model}

\subsection{Model formulation}

For the benefit of the reader, let us recall here the basic model formulated in [8]. It consists of a diseaseexposed population, described by a density function $n(a, t)$. The total population size is given by the following integral:

$$
N(t)=\int_{0}^{\infty} n(a, t) d a .
$$

We denote with $i(a, t), r(a, t)$ and $s(a, t)$ the nonnegative densities of the three epidemiological classes, respectively the infected, the removed and the susceptible individuals. We also assume that transitions between the classes are possible: in fact, by contagion a susceptible migrates into the class of infected; once identified as diseased, infect individuals will be immediately removed and considered to belong to the class $r$; finally, removed individuals can recover the disease and thus return into the class of susceptibles. This occurs because in this model we assume no disease-immunity.

The density $i(a, t)$ describes how many individuals of age $a$ are infected at time $t$. Its evolution is described by the following hyperbolic differential equation:

$$
\frac{\partial i}{\partial a}+\frac{\partial i}{\partial t}+(g+m(a, N)) i(a, t)-p(a) s(a, t) \widetilde{I}(t)=0
$$

where $m(a, N)$ denotes the biological mortality of the population under study, not due to the effect of epidemics, and $g$ denotes the rate at which individuals are identified as disease-carriers and therefore migrate into the class of removed individuals. Here $\widetilde{I}(t)=\int_{0}^{\infty} i(a, t) d a$ denotes the total population of infected individuals at time $t$ and $p(a)$ indicates the contagion rate between a susceptible aged $a$ and one infected. The last term in (2.2) therefore describes those individuals who migrate from class $s$ to class $i$.

As for individuals in the class of removed, the possible outcomes are either the recovery with subsequently the termination of the immunity period or the death either due to natural causes or possibly epidemics-related. In order to write down the equation that describes evolution of $r(a, t)$, we have also to consider the infected individuals aged $a$ at time $t$ recognized as diseased:

$$
\frac{\partial r}{\partial a}+\frac{\partial r}{\partial t}+(q(a)+z(a)+m(a, N)) r(a, t)-g i(a, t)=0
$$

Here $q$ denotes the recovery rate from the disease, $z$ represents the disease-related mortality.

We have now to state the equation concerning the class of susceptibles. An individual leaves this class in case of natural death or because of the contraction of the disease. This depends on the rate at which a susceptible individual has contacts with an infected one. At the same time, individuals that ended their immunity period after the disease, migrate from the class of removed into the class of susceptibles. Taking into account these facts, the equation for $s(a, t)$ is:

$$
\frac{\partial s}{\partial a}+\frac{\partial s}{\partial t}+s(a, t)(m(a, N)+p(a) \widetilde{I}(t))-q(a) r(a, t)=0 .
$$


In order to complete the description of the system dynamics, we have still to add another equation describing the reproduction of the population. Le us assume that only susceptible individuals reproduce and every newborn is susceptible himself. We have then:

$$
n(0, t)=s(0, t)=\int_{0}^{\infty} b(a, N) s(a, t) d a
$$

where $b(a, N)$ represents the reproduction function, also called maternity function. Note that the boundary conditions for the remaining unknowns are homogeneous, since no newborn is sick nor removed, $i(0, t)=r(0, t)=0$. Furthermore, to simplify the subsequent numerical simulations, a maximum age $A_{M A X}$ is fixed, at which individuals are assumed to be all dead.

Finally, the initial conditions of the problem are stated:

$$
i(a, 0)=I^{0}(a), \quad r(a, 0)=R^{0}(a), \quad s(a, 0)=S^{0}(a) .
$$

\subsection{Numerical simulations}

In order to perform numerical simulations, we take the maximum age to be $A_{M A X}=110$ and explicitly define the form of the other functions that play an essential role in the model. For this task, some euristics is employed. The disease-recovery function $q=q(a)$, i.e. the transition rate from $r$ to $s$, is chosen to have a maximum at $a \cong 37$ and to decrease as a function of $a$ in the following fashion:

$$
q(a)=\frac{C_{q}}{131}\left[\sin \left(\frac{a+10}{30}\right)+0.76\right]
$$

The function $p=p(a)$, i.e. the contact rate between a susceptible aged $a$ and an infected, should have a maximum for an intermediate age, corresponding to the age at which individuals work and therefore are most exposed to contacts with their similar. In fact, contacts of very young children in pre-schooling age and elderly people are certainly more restricted to their familiar entourage. The function is then taken as the following concave parabola

$$
p(a)=-C_{p} \frac{a(a-110)}{2218330} .
$$

To define the maternity function $b(a, N)$ and the natural mortality function $m(a, N)$, we collected available data, [5] and chose the following mathematical functions that adequately approximate these trends:

$$
b(a, N)=C_{b} \frac{1}{\sqrt{(50 \pi)}} e^{-\frac{(a-34)^{2}}{50}} N, \quad m(a, N)=C_{m} \frac{e^{0.007(a-30)}}{400} N .
$$

Also the mortality due to epidemics is supposed to be more likely for children and old people. Thus the disease-related mortality is empirically chosen to be:

$$
z(a)=C_{z}\left(-6.224 \cdot 10^{-7} a^{3}+1.521 \cdot 10^{-4} a^{2}-0.006944 a+0.10326\right) .
$$

With this background, we ran extensive computer simulations to understand the dynamic system's behaviour. To this end, we have used different programs, one based on the explicit Wendroff scheme [7], another one on the implicit Kwon-Cho method [4]. In this way we could validate the simulations, since their results agree, in spite of being obtained via different algorithms.

By suitably changing the coefficients of above defined functions, is possible to show different behaviours of the system's solutions. The total population of the three classes, susceptible, removed and infected, exhibit various features as functions of time. These are now summarized below, in Fig.s 1-5.

In the first situation, we observe the extinction, in few years, of the whole population, see Fig. 1. This is certainly not a desirable result. 


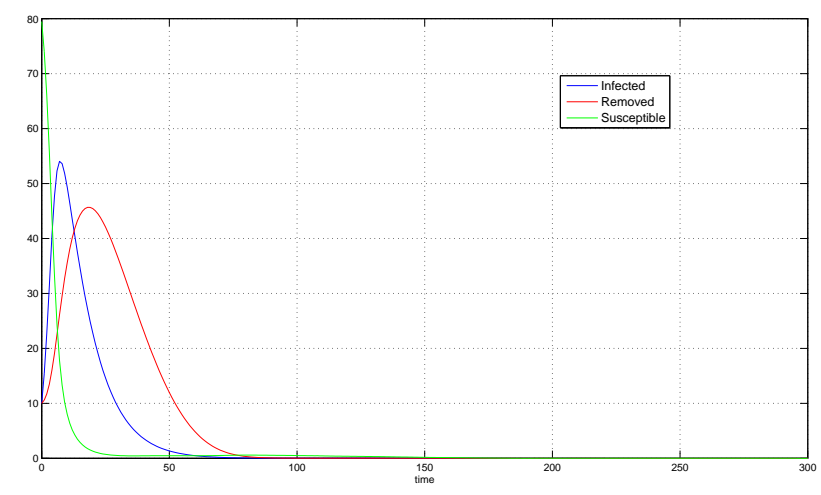

FiguRE 1 . The population become extinct with the following parameter choice: $C_{b}=$ 1.005, $C_{m}=0.2, C_{p}=0.9, C_{q}=0.01, C_{z}=0.9, g=0.09$.

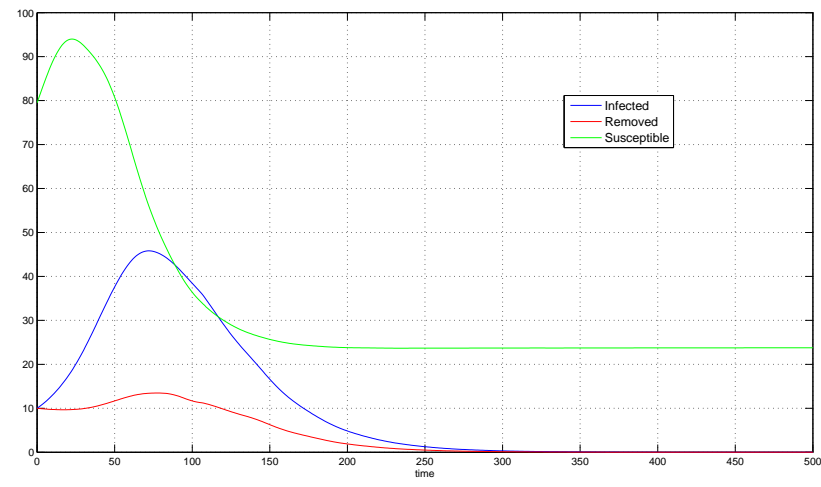

Figure 2. Disease eradication is obtained with the following parameters: $C_{b}=1.005$, $C_{m}=0.1, C_{p}=0.05, C_{q}=1, C_{z}=0.01, g=0.01$.

The second case is represented by the extinction of the disease, while stabilization for the number of individuals in the class of susceptible is achieved, see Fig. 2. This from the epidemiological point of view could be a goal to strive for.

The system can also coexist at a stable equilibrium, see Fig. 3.

For the last choice of parameters the sytsem exhibits periodic solutions for all the three classes of individuals, see Fig. 4. We have therefore a limit cycle in the three dimensional phase space, see Fig. 5.

It is also interesting to observe that for the same choice of parameters, the whole population shows persistent oscillations, Fig. 6 .

The numerical experiments have been performed with the following choice of the initial conditions:

$$
\begin{aligned}
i(a, 0) & =\frac{\left(e^{-\frac{a}{40}} \sin ^{2} \frac{a}{8}\right)-\frac{a}{10000}(a-105,05)}{3.761}, \\
r(a, 0) & =\frac{\left(e^{-\frac{a}{20}} \sin ^{2} \frac{a}{10}\right)-\frac{a}{100000}(a-110)}{1.158}, \\
s(a, 0) & =\frac{e^{-\frac{a}{10}}\left[\left(\frac{a}{10}\right)^{3}+1\right]+1}{0.925(a+1)} .
\end{aligned}
$$




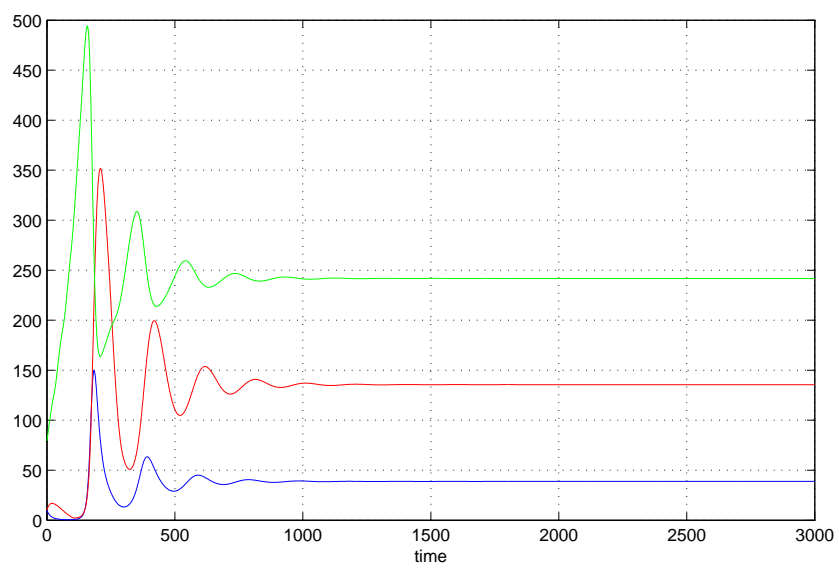

Figure 3 . The populations coexist at a stable equilibrium for the following parameters: $C_{b}=1.5, C_{m}=0.1, C_{p}=0.05, C_{q}=1, C_{z}=0.01, g=0.1$

We have also tried different choices of such functions, in particular constant initial conditions. However, with these changes the system evolution preserves the same trends.

\subsection{Bifurcation analysis}

In view of the fact that there is a transition in the stability of the coexistence equilibrium, we investigated the model parameters to understand which one is more responsible for this change. It turns out that the parameter that influences more the dynamical system's behavior is the coefficient $C_{b}$ multiplying the maternity function. We therefore exhibit a bifurcation diagram, Fig. 7, in order to understand how the system changes under the influence of $C_{b}$, letting it vary in the interval $[0.5,2.5]$.

For values of $C_{b} \leq 1$ the unique equilibrium of the system entails extinction of all the three populations. Increasing the parameter, at first the system attains a stable equilibrium with different values for each population. Past the bifurcation value at about $C_{b}=1.6$ it assumes a periodic behavior with increasing amplitudes as $C_{b}$ increases.

\section{Age- and stage-dependent epidemic model}

\subsection{Model set up.}

Let us consider now a stage structure also for the disease, introducing for an infected individual a parameter $w$ indicating the units of time since the contagion has occurred. A corresponding parameter for an individual in the class of removed denotes the units of time since the disease has been discovered and therefore cures began. The densities of these two classes become therefore functions of three variables, $i(a, w, t), r(a, w, t)$ while for the susceptibles we still have $s(a, t)$.

The total population in the class of removed individuals, for a specified age $a$ and a time $t$, is given by:

$$
R(a, t)=\int_{0}^{\infty} r(a, w, t) d w
$$

and a similar expression holds for the infected individuals:

$$
I(a, t)=\int_{0}^{\infty} i(a, w, t) d w .
$$



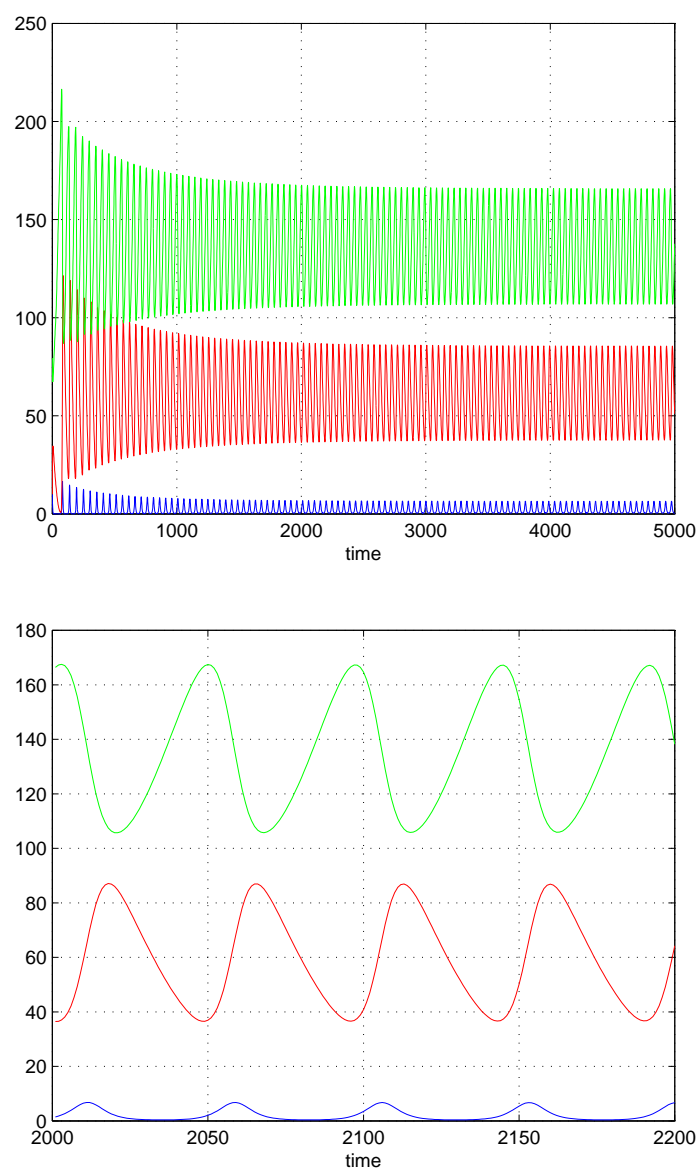

FigurE 4. Left: all subpopulations coexist via sustained oscillations, for the parameters $C_{b}=1.8, C_{m}=0.11, C_{p}=1.2, C_{q}=0.01, C_{z}=0.71, g=1.3$. Right: a blow up of the figure on the left.

Observe that for $w \geq a$ the above densities are identically zero, in view of the very definition of the model. The equation describing the evolution of $i(a, w, t)$ is now the following:

$$
\frac{\partial i}{\partial a}+\frac{\partial i}{\partial w}+\frac{\partial i}{\partial t}+(g(w)+m(a, N)) i(a, w, t)=0
$$

where $g=g(w)$ is here a function of the stage of the disease, representing the rate at which an individual in the stage $w$ of the disease is recognized as infected and thus removed from circulation. Moreover this function allows us to define the density of removed individuals at the initial stage of the recovery period:

$$
r(a, 0, t)=\int_{0}^{\infty} g(w) i(a, w, t) d w .
$$

As for the class of removed individuals, the governing equation now becomes:

$$
\frac{\partial r}{\partial a}+\frac{\partial r}{\partial w}+\frac{\partial r}{\partial t}+(q(a, w)+z(a, w)+m(a, N)) r(a, w, t)=0
$$




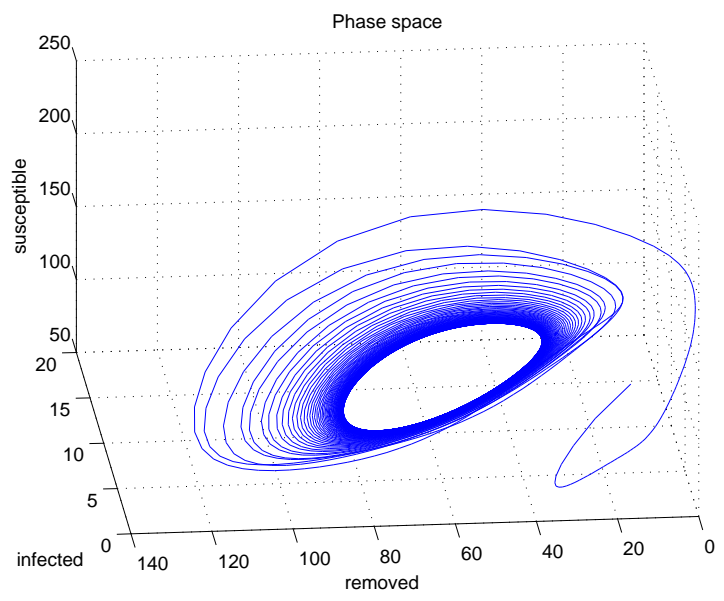

Figure 5. For the same parameters of Fig. 4 the limit cycle is shown in the phase space.

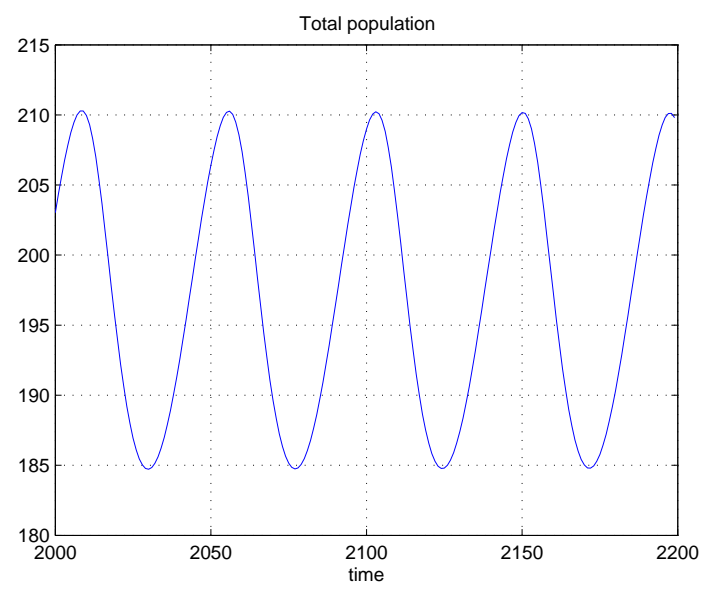

FiguRE 6. For the same parameters of Fig. 4 the total population shows stable persistent oscillations.

where in this case the disease recovery rate and the mortality due to the epidemics depend both on $a$ as well as on $w$. Furthermore, the introduction of the stage dependency, changes the evolution equation of the susceptibles $s$ as follows

$$
\frac{\partial s}{\partial a}+\frac{\partial s}{\partial t}+s(a, t)\left(m(a, N)+\int_{0}^{\infty} p(a, w) \widetilde{I}(w, t) d w\right)-\int_{0}^{\infty} q(a, w) r(a, w, t) d w=0
$$

where $\widetilde{I}(w, t)=\int_{0}^{\infty} i(a, w, t) d a$. Note that $p(a, w)$ indicates the contagion rate between one susceptible aged $a$ and an infected at stage $w$. It also helps to determine the infected individuals at stage zero of the disease:

$$
i(a, 0, t)=s(a, t) \int_{0}^{\infty} p(a, w) \widetilde{I}(w, t) d w .
$$




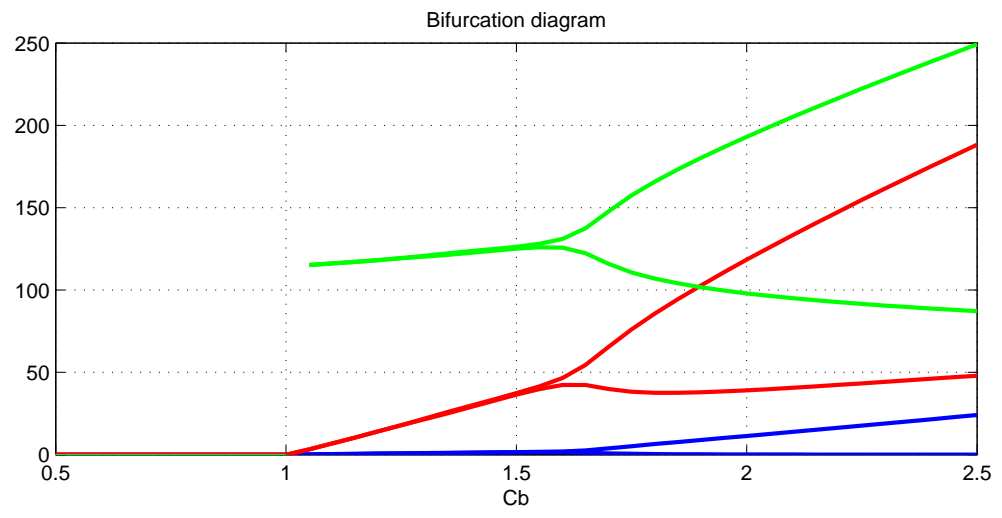

Figure 7. Bifurcations for the age-dependent model as functions of the parameter $C_{b}$.

The condition for newborns is the same as for the age-structured model, while the other boundary conditions become:

$$
\begin{array}{r}
s\left(A_{M A X}, t\right)=i\left(A_{M A X}, w, t\right)=r\left(A_{M A X}, w, t\right)=0, \\
i\left(a, W_{M A X}, t\right)=r\left(a, W_{M A X}, t\right)=0,
\end{array}
$$

where $W_{M A X}$ represents the maximum stage allowed, at which individuals are supposed to have all left their respective classes.

Finally, we report the initial conditions

$$
i(a, w, 0)=I^{0}(a, w) \quad a>w, \quad r(a, w, 0)=R^{0}(a, w) \quad a>w, \quad s(a, 0)=S^{0}(a),
$$

where $S^{0}(a), I^{0}(a, w)$ and $R^{0}(a, w)$ are nonnegative given functions.

\subsection{Numerical simulations}

As done for the previous model let us now explicitly define the functions involved in the system formulation. Firstly, the function $g=g(w)$, i.e. the rate at which an individual at stage $w$ of the disease is recognized as carrier and migrates into the class of removed, is chosen to be a linearly increasing function of the stage:

$$
g(w)=2 C_{g} w
$$

We consider next the recovery rate of the disease $q=q(a, w)$; as a function of $a$ it is a parabola, while it decreases with $w$ :

$$
q(a, w)=C_{q} \frac{1}{41.6639}\left[\sin \left(\frac{a+10}{30}\right)+0.76\right] e^{-3 w} .
$$

The contagion rate between a susceptible aged $a$ and an infected in stage $w$, namely $p=p(a, w)$, is larger for young and middle aged people and is assumed to decrease with respect to the stage $w$ :

$$
p(a, w)=C_{p} \frac{-a(a-110) e^{-4 w}}{54442.57852} .
$$

As for the maternity and biological mortality functions $b(a, N)$ and $m(a, N)$, we use the same functions defined for the age-structured model, because they are obviously independent on $w$. Concerning the disease-related mortality $z=z(a, w)$, we assume it to be higher for children and old people and for advanced stages of the disease:

$$
z(a, w)=2 C_{z} \frac{\left(-6.224 \cdot 10^{-7} a^{3}+1.521 \cdot 10^{-4} a^{2}-0.006944 a+0.10326\right) w}{14.08121} .
$$


By suitably changing the coefficients of the above defined functions, as done in the previous model, it is possible to obtain different behaviours for the total population of the three classes with respect to the time $t$.

The numerical method used for the simulations reported in the Fig.s 8-10 is an extension of the well-known Wendroff implicit scheme, see [7].

The first situation that we observe, Fig. 8, represents the extinction of the two disease-affected populations, namely infected and removed individuals, while the population of susceptibles keeps growing:

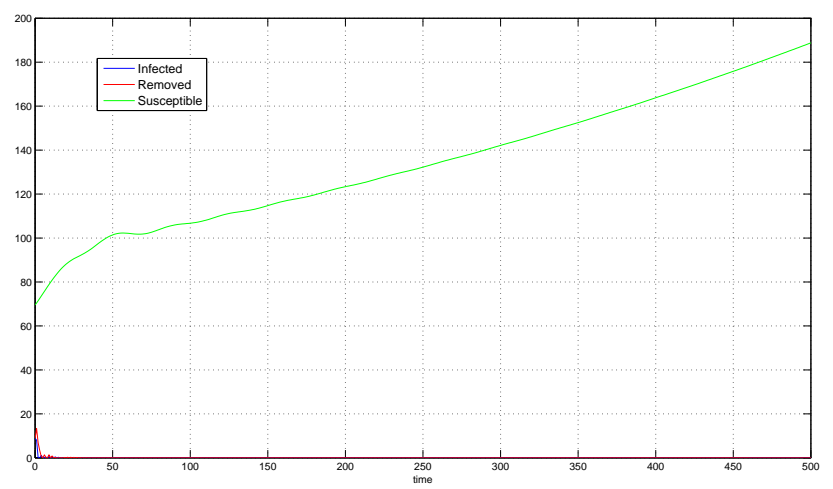

FiguRE 8. Disease extinction and growth of the susceptible population, for the parameter values $C_{b}=1.065, C_{m}=0.29, C_{p}=0.01, C_{q}=0.1, C_{z}=0.01, C_{g}=1.6$.

In the second case, similarly as to what happens for the age-structured model, we can find a set of parameters for which the system shows a periodic trend for all the three populations, Fig. 9. This means that the system trajectories evolve toward a limit cycle. The latter appears clearly in the phase space diagram of Fig. 10.

Again, for the same choice of parameters, we find persistent oscillations for the whole population, Fig. 11.

The simulations have been obtained with the following initial conditions:

$$
\begin{aligned}
i(a, w, 0) & =\frac{\left(e^{-\frac{a}{40}} \sin ^{2} \frac{a}{8}\right)-\frac{a}{10000}(a-105,05)}{2.5073} e^{-4 w} \\
r(a, w, 0) & =\frac{\left(e^{-\frac{a}{20}} \sin ^{2} \frac{a}{10}\right)-\frac{a}{100000}(a-110)}{0.772} e^{-4 w} \\
s(a, 0) & =\frac{e^{-\frac{a}{10}}\left[\left(\frac{a}{10}\right)^{3}+1\right]+1}{1.0571(a+1)}
\end{aligned}
$$

but, as in the previous model, running the codes with different choices for these functions, such as for instance constant initial conditions, these changes do not influence the final outcome of the system trajectories.

\subsection{Bifurcations for the age- and stage-dependent model}

We finally investigated the nature of the Hopf bifurcation numerically. Again, as in the previous model of Section 2, we identify a bifurcation parameter, in this case it is $C_{b}$, and provide in Fig. 12 the bifurcation diagram. It is observed that for $C_{b}<1$ the disease is eradicated, for $1<C_{b}<1.3$ the disease becomes 

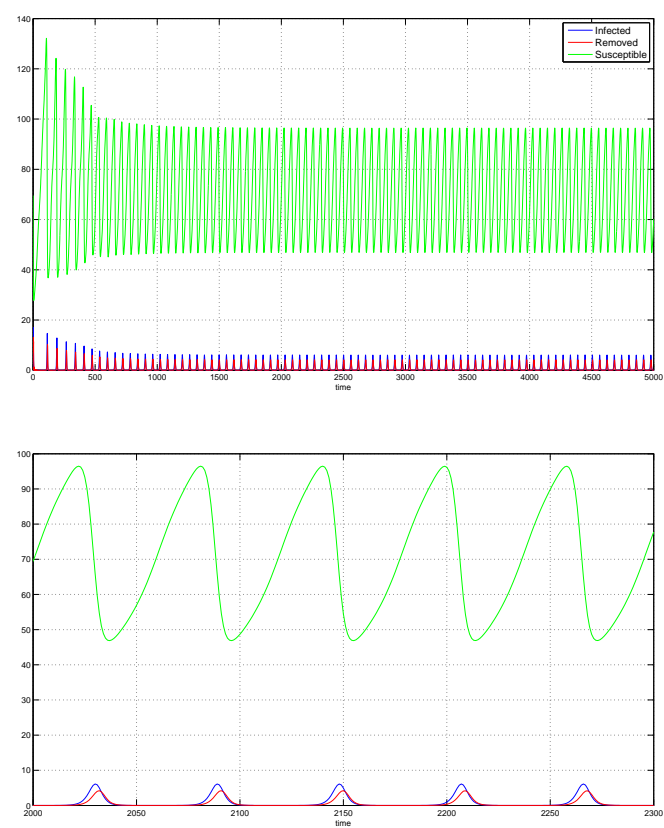

Figure 9. Coexistence of all the populations, with endemic disease, through persistent oscillations, for the parameter values $C_{b}=1.5, C_{m}=0.11, C_{p}=1.2, C_{q}=0.01$, $C_{z}=0.71, C_{g}=1.3$.

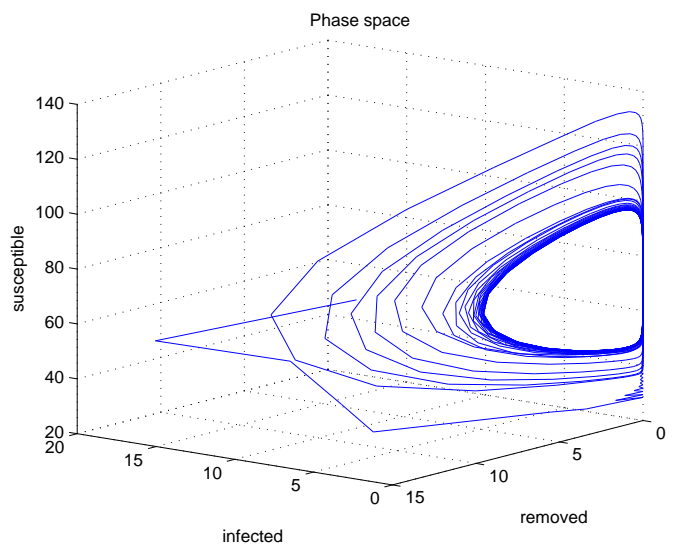

FiguRE 10. Phase space representation of the limit cycle, for same the parameter values of Fig. 9.

endemic with a stable coexistence equilibrium, and finally for larger values this equilibrium destabilizes and limit cycles of wider and wider amplitudes arise. 


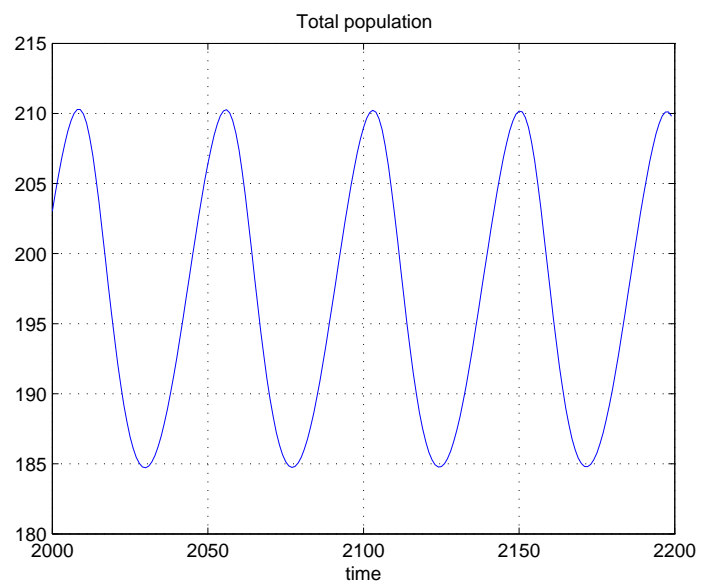

FiguRE 11. For the same parameters of Fig. 9 the total population shows once again stable persistent oscillations.

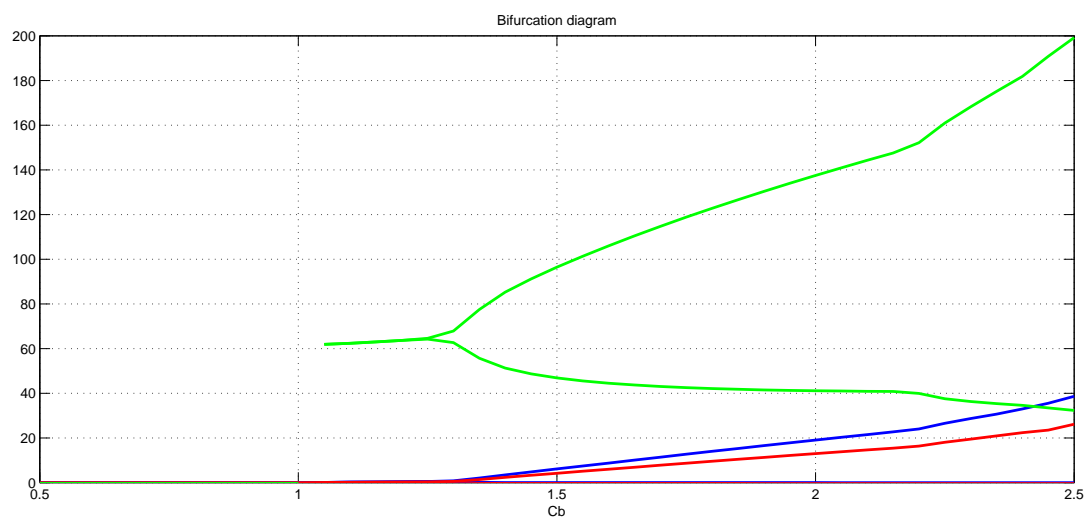

Figure 12. Bifurcations for the age-dependent model in terms of the bifurcation parameter $C_{b}$.

\section{Discussion}

The models introduced here are of rather theoretical nature. We do not focus our attention on any particular disease, except for it being transmissible by contacts among infected and susceptible individuals. The structuring both of the population via age cohorts and of the disease through its "age" since infection, namely the stage of the disease, allow the onset of persistent oscillations in the classes partitioning the whole population. These limit cycles arise in the systems presented here, while the classical SIR epidemics model does not allow them. Mathematically this is due to the more complex way the interactions are described. From the applied point of view, recurrences of epidemics are well known. For instance, the classical case of measles, which has been monitored now for almost a century in the UK, shows periodic outbreaks with a period of two years. The models presented here could suggest that such occurrences might be due to the intrinsic nature of the population structure, or to the way the disease progresses in its evolution within infected individuals, rather than to external causes, for instance due to 
forcing functions expressing e.g. seasonality. This is certainly not a conclusive assertion, but rather an indication of possible future speculation and validation.

\section{References}

[1] L. M. Abia, O. Angulo, J. C. López-Marcos. Age-structured population models and their numerical solution. Ecological Modelling, 188 (2005), 122-123.

[2] M. Haque, E. Venturino. The role of transmissible diseases in Holling-Tanner predator-prey model. Theoretical Population Biology, 70 (2006), 273-288.

[3] H. W. Hethcote. The mathematics of infectious diseases. SIAM Review, 42 (2000), 599-653.

[4] Y. Kwon, C. K. Cho. Second-order accurate difference methods for a one-sex model of population dynamics. SIAM J. Numer. Anal, 30 (1993), 1385-1399.

[5] http//demo.istat.it

[6] H. Malchow, S. Petrovskii, E. Venturino. Spatiotemporal patterns in Ecology and Epidemiology. CRC, Boca Raton, 2008.

[7] G. D. Smith. Numerical Solution of Partial Differential Equations: Finite Difference Methods. third ed. Clarendon press, Oxford, 1985.

[8] E. Venturino. A generalization of the classical epidemiology model. IMACS Transactions on Scientific Computation '85, vol. 5 - Modelling of Biomedical Systems, (1985), 243-248.

[9] E. Venturino. A stage-dependent ecoepidemic model. WSEAS Transactions on Biology and Biomedicine, 1 (2004), 449-454.

[10] E. Venturino. Ecoepidemiology 15 years later: a review. Numerical Analysis and Applied Mathematics, T. Simos (Editor), Proceedings of ICNAAM 2007, AIP 936, (2007) 31-34. 